M2 macrophage polarisation is associated with alveolar formation during postnatal lung development

Jones et al.

() Biomed Central 


\title{
M2 macrophage polarisation is associated with alveolar formation during postnatal lung development
}

\author{
Christina V Jones ${ }^{1}$, Timothy M Williams ${ }^{1}$, Kenneth A Walker ${ }^{1}$, Hayley Dickinson², Samy Sakkal ${ }^{1}$, Bree A Rumballe ${ }^{3}$,
} Melissa H Little ${ }^{3}$ Graham Jenkin ${ }^{2}$ and Sharon D Ricardo ${ }^{1 *}$

\begin{abstract}
Background: Macrophages are traditionally associated with inflammation and host defence, however a greater understanding of macrophage heterogeneity is revealing their essential roles in non-immune functions such as development, homeostasis and regeneration. In organs including the brain, kidney, mammary gland and pancreas, macrophages reside in large numbers and provide essential regulatory functions that shape organ development and maturation. However, the role of macrophages in lung development and the potential implications of macrophage modulation in the promotion of lung maturation have not yet been ascertained.
\end{abstract}

Methods: Embryonic day (E)12.5 mouse lungs were cultured as explants and macrophages associated with branching morphogenesis were visualised by wholemount immunofluorescence microscopy. Postnatal lung development and the correlation with macrophage number and phenotype were examined using Colonystimulating factor-1 receptor-enhanced green fluorescent protein (Csf1r-EGFP) reporter mice. Structural histological examination was complemented with whole-body plethysmography assessment of postnatal lung functional maturation over time.

Flow cytometry, real-time (q)PCR and immunofluorescence microscopy were performed to characterise macrophage number, phenotype and localisation in the lung during postnatal development. To assess the impact of developmental macrophage modulation, CSF-1 was administered to neonatal mice at postnatal day (P)1, 2 and 3, and lung macrophage number and phenotype were assessed at P5. EGFP transgene expression and in situ hybridisation was performed to assess CSF-1R location in the developing lung.

Results: Macrophages in embryonic lungs were abundant and densely located within branch points during branching morphogenesis. During postnatal development, structural and functional maturation of the lung was associated with an increase in lung macrophage number. In particular, the period of alveolarisation from P14-21 was associated with increased number of Csf1r-EGFP+ macrophages and upregulated expression of Arginase 1 (Arg1), Mannose receptor 1 (MrC1) and Chemokine C-C motif ligand 17 (CCl17), indicative of an M2 or tissue remodelling macrophage phenotype. Administration of CSF-1 to neonatal mice increased trophic macrophages during development and was associated with increased expression of the M2-associated gene Found in inflammatory zone (Fizz) 1 and the growth regulator Insulin-like growth factor (Igf)1. The effects of CSF-1 were identified as macrophagemediated, as the CSF-1R was found to be exclusively expressed on interstitial myeloid cells.

(Continued on next page)

\footnotetext{
* Correspondence: Sharon.Ricardo@monash.edu

'Department of Anatomy and Developmental Biology, Monash University,

Clayton, Victoria, Australia

Full list of author information is available at the end of the article
}

\section{Biomed Central}

(c) 2013 Jones et al.; licensee BioMed Central Ltd. This is an Open Access article distributed under the terms of the Creative Commons Attribution License (http://creativecommons.org/licenses/by/2.0), which permits unrestricted use, distribution, and reproduction in any medium, provided the original work is properly cited. 


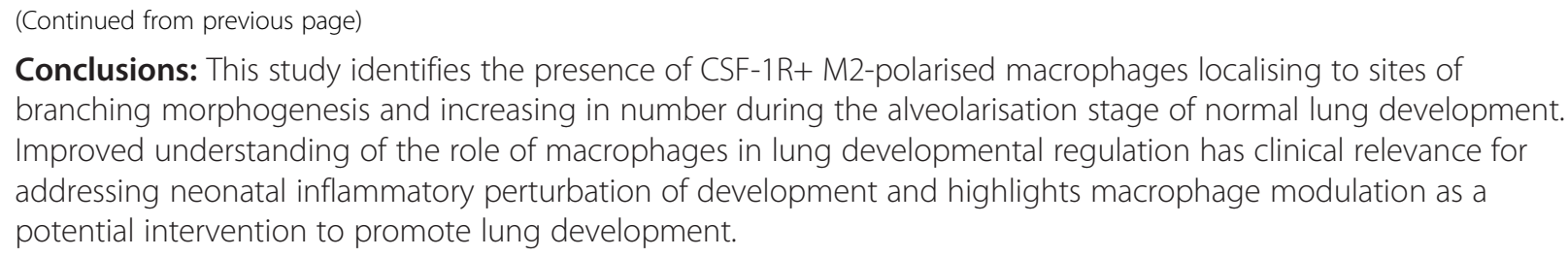

Keywords: Macrophage, CSF-1, Lung development, M2, Alveolarisation

\section{Background}

A diverse network of regulators govern the developmental transformation from multipotent progenitors in the post-induction lung buds to the complex architecture and highly specialised terminal cell types that make up the mature lung. These include a range of growth factors, signalling pathways and transcriptional regulators that arise from epithelial, mesodermal and mesothelial origins [Reviewed in [1]]. Another important component of the lung organogenic milieu is the tissue macrophage. Traditionally associated with host defence, inflammation and scavenging functions, a greater appreciation of macrophage diversity has revealed broader functions of macrophages including vital roles in tissue repair [2-6] and organ development [7-11].

Macrophages first arise in the yolk sac around embryonic day (E) 8 in the mouse, and migrate into the developing head before colonising the entire embryo [12-14]. Large numbers of macrophages are present in virtually all developing organs, with maximum numbers correlating with key periods of organogenesis [15]. Macrophages contribute to development through apoptosis, phagocytic clearance of cellular debris associated with tissue remodelling, and as potent effector cells producing a range of trophic factors that stimulate growth, regulate cellular differentiation and promote angiogenesis [Reviewed in [16]]. Furthermore, mice deficient in tissue macrophages display a range of developmental abnormalities including skeletal and neurological deficiencies and impaired growth and fertility [17-19].

Macrophages are essential in the normal development of the mammary gland, pancreas and kidney; organs which, similar to the lung, develop through branching morphogenesis. Normally, macrophages are located surrounding developing terminal buds but, in their absence, branching is impaired resulting in atrophic, poorlybranched terminal buds in the mammary gland $[9,20]$, and abnormal islet cell morphology and reduced insulin production in the pancreas [21,22]. Furthermore, the addition of the key macrophage regulatory cytokine colony-stimulating factor (CSF)- 1 to embryonic organ cultures was shown to enhance development of the pancreas [8] and kidney [11], which was associated with increased number of tissue macrophages. While the organogenic contribution of macrophages to these organs is well described, less has been investigated regarding their roles in the development of the lung.

Macrophages are present in the lung from the initiation of development, and at E10 are located abundantly in the mesenchyme and in association with elongating lung buds $[23,24]$. Fetal lung macrophages likely contribute to lung development through the regulation of apoptosis and clearance of cellular debris. Defective pulmonary phagocytosis in the phosphatidylerine receptor $(p s r)^{-/-}$ mutant mouse is associated with impaired removal of apoptotic cells during development, which in turn results in solid lungs devoid of alveoli [25]. Macrophages in the lung are also sources of trophic factors such as insulin-like growth factor (IGF)-1 [26] and wingless-type MMTV integration site (Wnt)7b [27], both of which are important regulators in lung development.

To date, the understanding of lung macrophage function has focussed on pathological implications in settings associated with neonatal inflammation with little insight regarding their contribution to normal developmental regulation. In this study, we provide the first report characterising macrophages during the alveolarisation stage of lung development in the mouse. Macrophages in the postnatal lung displayed a phenotype indicative of an M2 or alternatively activated macrophage polarisation state, which is characteristic of macrophages involved in trophic and tissue remodelling functions. Furthermore, the number of CSF-1 receptor $(\mathrm{CSF}-1 \mathrm{R})+\mathrm{F} 4 / 80+$ macrophages was increased during alveolarisation and, together with the expression of M2-associated genes, indicates the importance of trophic macrophages during this period of significant tissue remodelling.

\section{Methods}

\section{Animals}

All animal experiments were approved in advance by the Monash University Animal Ethics Committee and conducted in accordance with the "Australian Code of Practice for the Care and Use of Animals for Scientific Purposes" ( $7^{\text {th }}$ Edition, 2004). For embryonic lung culture, time-mated C57BL/6J females were humanely euthanised by cervical dislocation at 12.5 days postcoitum, with 0.5 defined as noon on the day a 
plug was detected. Embryos were collected, development was assessed using the Theiler Staging (TS) criteria (TS 1516/27-31 somites) and the lungs were dissected. Postnatal lung analyses were performed on Csf1r-EGFP mice, which directs enhanced green fluorescent protein (EGFP) expression to cells of the myeloid lineage under the control of the Csf1r promoter [14]. Neonatal mice were administered mouse recombinant CSF-1 ( $1 \mu \mathrm{g} / \mathrm{g}$ bodyweight; University of Queensland Protein Facility, Brisbane, Australia) in phosphate buffered saline (PBS) via intraperitoneal (i.p.) injection at a final volume of $50 \mu \mathrm{l}$ at postnatal day (P) 1, 2 and 3, with P1 defined as day of birth [2]. Littermate controls received vehicle PBS at the equivalent final volume.

\section{Embryonic lung culture and wholemount immunofluorescence labelling}

Embryonic lungs were transferred onto polycarbonate membranes ( $3 \mu \mathrm{m}$ pore size; GE Water and Processing Technologies, Oakville, Canada), floating on serum-free media in a 24 well plate (BD Biosciences). Culture media was composed of Dulbecco's Modified Eagle Medium F/12 (Gibco/ Invitrogen, Mulgrave, VIC, Australia), supplemented with $2.5 \mathrm{mM}$ L-glutamine (Gibco/Invitrogen), $5 \mu \mathrm{g} / \mathrm{ml}$ insulin transferrin selenium (Gibco/Invitrogen) and $100 \mu \mathrm{g} / \mathrm{ml}$ penicillin streptomycin (Gibco/Invitrogen). Organs were incubated for 48 hours at $37^{\circ} \mathrm{C}$ in $5 \% \mathrm{CO}_{2}$. Explants were fixed in ice-cold methanol (for $30 \mathrm{~min}$ at $-20^{\circ} \mathrm{C}$ ) and wholemount immunolabelled to visualise macrophages in development. Explants were permeablised in $0.1 \%$ Triton X (in PBS for $10 \mathrm{~min}$ ) and non-specific binding was blocked by incubation with $10 \%$ goat serum and $2 \%$ bovine serum albumin (BSA; in PBS for $30 \mathrm{~min})$. Explants were incubated with rat antiF4/80 (1:100; Serotec, Kidlington, UK; Clone Cl:A3-1) and rabbit anti-E-cadherin (1:100: Cell Signalling Technologies, Danvers, MA, USA; Clone 24E10) primary antibodies (at $37^{\circ} \mathrm{C}$ for $2 \mathrm{hrs}$ ) to demarcate macrophages and lung epithelium, respectively. Explants were washed in PBS $(3 \times 5 \mathrm{~min}$ at room temperature), incubated with Alexa Fluor ${ }^{\circledR}$ goat anti-rat 555 and goat anti-rabbit 488 (Invitrogen; 1:500) secondary antibodies (at $37^{\circ} \mathrm{C}$ for $1 \mathrm{hr}$ ). Membrane-bound explants were placed on glass slides with PBS and coverslipped.

\section{Postnatal lung histology and macrophage immunofluorescence labelling}

Lungs were reinflated and fixed in situ through intratracheal instillation of $10 \%$ buffered formalin at a pressure of 20 $\mathrm{cmH}_{2} \mathrm{O}$. After ligating the trachea, the entire thorax was immersion fixed for $24 \mathrm{hr}$ before lungs were dissected. To assess histology, lungs were processed, embedded in paraffin wax, sectioned at $5 \mu \mathrm{m}$, mounted on Polylysine ${ }^{\mathrm{TM}}$ slides (Menzel-Glaser, Braunschweig, Germany) and stained with haematoxylin and eosin. For immunofluorescence labelling, excised lungs were placed in 30\% sucrose solution (in PBS) and allowed to infiltrate overnight at $4^{\circ} \mathrm{C}$. Organs were immersed in OCT compound (Sakura, Torrance, CA, USA) in Tissue-Tek ${ }^{\circledR}$ cryomoulds (Sakura) and frozen by floating moulds on chilled isopentane on dry ice. Lungs were cryosectioned at $5 \mu \mathrm{m}$ and mounted on SuperFrost ${ }^{\circledR}$ Plus slides (Menzel-Glaser). For macrophage visualisation, sections were blocked in $10 \%$ goat serum, incubated with rat anti-F4/80 primary antibody (1:100; Serotec), washed and incubated with AlexaFluor ${ }^{\circledR}$ goat anti-rat 488 secondary antibody (1:500; Invitrogen). Sections were counterstained with DAPI nuclear stain (1:10,000 in PBS; Invitrogen) for 5 minutes, washed, mounted with DAKO fluorescent mounting medium (DAKO Cytomation, Botany, NSW, Australia) and coverslipped.

\section{Flow cytometry}

Whole lungs underwent enzymatic and mechanical digestion to yield a single cell suspension as described previously [2]. In brief, organs were finely minced and incubated in $1 \mathrm{ml}$ digestion buffer; comprising $1 \mathrm{mg} / \mathrm{ml}$ collagenase/dispase (Roche Diagnostics, Indianapolis, IN, USA), 0.1\% DNase I (Roche Diagnostics) and $5 \mathrm{mM}$ $\mathrm{CaCl} 2$ in Hank's Balanced Salt Solution (Invitrogen) at $37^{\circ} \mathrm{C}$ for 20 minutes. Lungs were mechanically disrupted using a $1000 \mu \mathrm{l}$ pipette, before cells were gently passed through a 25-gauge needle to yield a single cell suspension. Cell suspensions were washed in fluorescence-activated cell sorting (FACS) buffer; comprising PBS supplemented with 0.2\% BSA, 0.5 M ethylenediaminetetraacetic acid (EDTA) and $0.02 \%$ sodium azide, and centrifuged at 485 relative centrifugal force (rcf; for 5 minutes at $4^{\circ} \mathrm{C}$ ). Red blood cells were lysed by resuspending samples in $1 \mathrm{ml}$ of red blood cell lysis buffer (at $37^{\circ} \mathrm{C}$ for $1 \mathrm{~min} ; 8.3 \mathrm{~g} / \mathrm{L}$ ammonium chloride; $\mathrm{pH}$ 7.5;) and cell suspensions were filtered through a $40 \mu \mathrm{m}$ cell strainer (BD Biosciences, North Ryde, NSW, Australia). Cell counts were performed using a Coulter $^{\circledR}$ Particle Count and Size Analyzer (Beckman Coulter Australia Pty Ltd, Gladesville, NSW, Australia). To assess macrophages across postnatal development, $1 \times 10^{6}$ cells were immunolabelled with anti-CD45 PE Cy5-conjugated antibody (1:1000; BD Biosciences; Clone 30-F11) at a final volume of $20 \mu \mathrm{l}$ for 20 minutes at $4^{\circ} \mathrm{C}$ in a 96 well plate. Cells were washed in FACS buffer and centrifuged, repeated twice, before being resuspended in $200 \mu \mathrm{I}$ FACS buffer and run on a BD FACSCalibur cytometer (BD Biosciences). To assess macrophages at P5 following CSF-1 administration, cells were immunolabelled with anti-CD45 APC Cy7-conjugated (1:800; BioLegend, San Diego, CA, USA; Clone 30-F11) and rat anti-F4/80 APC-conjugated (1:200; eBioscience, San Diego, CA, USA; Clone BM8) antibodies. Samples were run on a BD FACSCanto II cytometer (BD Biosciences). Data analysis was performed using Flow Jo FCS analysis software (Tree Star Inc., Ashland, OR, USA). 


\section{Plethysmography}

Respiratory physiology across a time course of postnatal development was assessed using unrestrained barometric whole-body plethysmography, as described previously $[28,29]$. In brief, mice were placed in a sealed cylindrical Perspex chamber (Neonate; $75 \mathrm{~mm} \times 50 \mathrm{~mm}$, Adolescent/Adult; $150 \mathrm{~mm} \times 50 \mathrm{~mm}$ ), where changes in pressure caused by breath tidal movements were measured using a volumetric pressure transducer (model PT5A; Grass Instrument Co., Quincy, MA, USA), amplified (Octal Bridge Amp model ML228 and Powerlab 8/30 model ML870; ADInstruments, Bella Vista, NSW, Australia) and the respiratory trace patterns recorded using Chart ${ }^{\mathrm{st}}$ software (v5.1; ADInstruments). At the beginning of each session the plethysmograph was calibrated by measuring the pressure deflection caused by the injection of a known volume $(300 \mu \mathrm{l})$ of air into the chamber. The temperature and relative humidity within the chamber were noted at the beginning and end of recordings (model HM34; Vaisala, Hawthorn, VIC, Australia). Waveform analysis (Chart ${ }^{\mathrm{tw}}$; ADInstruments) of respiratory traces was used to directly derive the pressure deflection per tidal breath $\left(\mathrm{P}_{\mathrm{T}}\right)$, total breath cycle time $\left(\mathrm{T}_{\text {tot }} ; \mathrm{sec}\right)$, breath frequency (f; breaths $\left./ \mathrm{min}\right)$, inspiration time $\left(\mathrm{T}_{\mathrm{i}} ;\right.$ sec $)$ and expiration time $\left(\mathrm{T}_{\mathrm{e}} ; \mathrm{sec}\right)$. To calculate tidal volume $\left(\mathrm{V}_{\mathrm{T}} ; \mathrm{mL}\right)$, the $\mathrm{P}_{\mathrm{T}}$ value obtained from the respiratory trace was inputted into the equation of Drorbaugh and Fenn [30], which was subsequently used to determine minute volume $\left(\mathrm{V}_{\mathrm{E}} ; \mathrm{mL} / \mathrm{min} ; \mathrm{V}_{\mathrm{T}} \times \mathrm{f}\right)$ and inspiratory flow rate $\left(\mathrm{V}_{\mathrm{T}} / \mathrm{T}_{\mathrm{i}} ; \mathrm{mL} / \mathrm{sec}\right)$.

\section{QPCR}

Semi-quantitative real-time (qPCR) was used to assess gene expression in whole lungs across postnatal development and in response to CSF-1. Lungs were dissected and snap frozen in RNAlater ${ }^{\circledR}$ RNA stabilisation reagent (Qiagen, Doncaster, VIC, Australia). Total RNA was extracted from organs using an RNeasy Mini Kit (Qiagen) and concentration and purity were analysed using a Nanodrop ${ }^{\circledR}$ Spectrometer (Nanodrop ${ }^{\circledR}$ Technologies, Wilmington, DE, USA). RNA was converted to cDNA using a High Capacity cDNA Reverse Transcription Kit (Applied Biosystems, Mulgrave, VIC, Australia). qPCR was performed using Taqman ${ }^{\circledR}$ Gene Expression Assays (Applied Biosystems) which provided pre-designed primer and probes to assess the genes $\beta$-actin (Actb; Assay ID: Mm00607939_s1), Chemokine C-C motif ligand (Ccl)2 (Mm00441242_m1), Inducible nitric oxide synthase (Nos2; Mm00440485_m1), Tumor necrosis factor- $\alpha$ (Tnf; Mm00443258_m1), Ccl17 (Mm00516136_m1), Arginase 1 (Arg1; Mm00475988_m1), Igf1 (Mm00439561_m1) and Found in Inflammatory Zone 1 (Fizz1; Mm00445109_m1). Reactions were performed in triplicate and run on a 7500 Real-Time PCR machine using SDS Software (v1.3; Applied Biosystems). Threshold cycle $(\mathrm{Ct})$ values were normalised against endogenous Actb expression and presented as relative quantification $(\mathrm{RQ})$.

\section{In situ hybridisation and microscopy}

Section in situ hybridisation for the Csf1r gene was performed on paraffin-embedded, $5 \mu \mathrm{m}$ sections of E12.5 embryonic lungs, as described previously [31] (Probe ID: MGI:50000914; http://www.gudmap.org). Sections were counterstained with haematoxylin. Light and fluorescence microscopy were performed using an Olympus Provis AX70 microscope (Olympus, Mt Waverley, VIC, Australia) and AnalysisB software (Soft Imaging Systems GmbH, Muenster, Germany). Bright field images were captured using a DP70 colour camera (Olympus). Fluorescence images were captured using an F-View black and white camera (Olympus). Image preparation and compilation was performed using AnalysisB software (Soft Imaging Systems) and Microsoft Power Point (Microsoft Corporation, Redmond, WA, USA).

\section{Statistical analysis}

Data is presented as mean \pm standard error of the mean (SEM). Statistical analysis was performed using GraphPad Prism $^{\text {Tw }}$ (Version 5 for Windows; GraphPad Software Inc, La Jolla, CA, USA). Significance was assessed using a oneway ANOVA and Tukey's post hoc test for comparisons across multiple time points or unpaired Student's $t$-test for comparisons between two experimental groups. A $\mathrm{p}$ value $<0.05$ was considered statistically significant.

\section{Results}

Macrophages are abundant in embryonic lungs and localise within branch points

To assess macrophages and their involvement in lung branching morphogenesis, E12.5 lungs were cultured as embryonic explants, with a continuation of branching morphogenesis observed over 48 hours of culture (Figure 1A-C). In this model system, flattening of the organ facilitated wholemount visualisation and the examination of macrophage localisation within the embryonic lung. Immunofluorescence labelling demonstrates that embryonic macrophages express the mature macrophage marker F4/80 and are found abundantly within embryonic lungs undergoing branching morphogenesis (Figure 1D\&E). In particular, the dense concentration of macrophages localised within branch points is prominent (Figure 1E). Such abundance, branch-specific location and intimate epithelial interaction support the relevance of macrophages in the regulation of lung development. Furthermore, this system indicates the importance of early fetal macrophages in colonising organs undergoing development. With the explant system eliminating the contribution of infiltrating cells at later stages of development, it also indicates that the large numbers of macrophages 


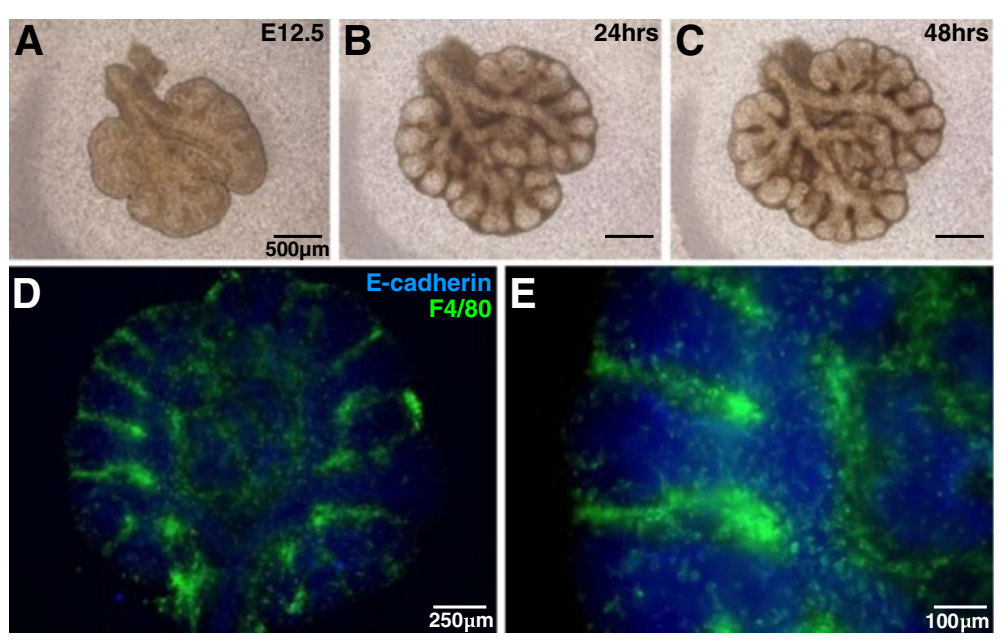

Figure 1 Macrophages are abundant in developing embryonic lungs. Ex vivo culture of E12.5 embryonic lungs, maintained for 48 hours on floating polycarbonate membranes at the air-liquid interface, supported continuation of branching morphogenesis (A-C). Wholemount immunofluorescence labelling of the lung epithelium (anti-E-cadherin; blue) and macrophages (anti-F4/80; green) revealed extensive macrophage accumulation within developing lungs (D), and in particular within branch points (E).

observed are seeded within the lung before E12.5, and are then maintained through local mechanisms to support ongoing branching morphogenesis.

\section{Macrophages are associated with the structural and functional postnatal maturation in the mouse}

In the mouse, the lung undergoes a significant period of postnatal development comprising both the closing stages of the saccular phase (E18.5-P5) and the alveolarisation phase (P5-P36) [1,32], and thereby provides an important animal species for investigating aspects of developmental regulation. The structural and functional maturation across the time course of postnatal lung development was characterised in Csf1r-EGFP mice.

Structural maturation facilitates the progressive gain in gas exchange efficiency; from large, thick-walled terminal sacs to smaller, thin-walled alveoli with a large surface area (Figure 2A-E). Histologically at P5, the lung parenchyma consisted of large terminal sacs (Figure 2A), and with continuing alveolarisation the subdivision into smaller alveoli through the process of secondary septation was evidenced by the formation of ridges on sac walls invading into the alveolar space (Figure 2B\&C). Continued secondary septation was evident at P14, with significant numbers of smaller alveoli present (Figure 2C). By P21 there was considerable thinning of alveolar walls bringing blood vessels into close association with the epithelium lining the alveolar space (Figure 2D). In these later stages of the alveolarisation phase, much of the secondary septation was complete and maturation involved thinning of the alveolar wall interstitium (Figure 2D). The adult lung at 3M showed all the structural hallmarks of an efficient gas exchange organ; large number of alveoli providing a large surface area and extremely thin walls to allow for efficient gas exchange (Figure 2E). Postnatal development was accompanied by the identification of macrophages in the lung parenchyma. Visualised by Csf1r-EGFP expression, these myeloid cells were predominantly macrophages, evident by their consistent co-expression of F4/80 (Figure 2F-J). The large numbers of macrophages within the lung from P5-P21 correlates with the key period of alveolar development.

These structural changes were mirrored by the functional improvements observed during postnatal development. Trace recordings from unrestrained barometric whole-body plethysmography illustrated the breath patterns and functional maturation of the lungs of mice during postnatal development through measurement of tidal pressure changes within the chamber due to respiration (Figure 2K-0). At P5, before alveolarisation, the respiratory capacity was limited, evidenced by trace recordings where breaths were shallow, uneven and dispersed (Figure 2K). With maturation and the formation of increasing numbers of alveoli from P7-P14 (Figure 2L\&M), breaths became deeper, more frequent and more even. At P21, a breath pattern comparable to the adult was observed (Figure 2N), however as lung growth continued tidal volume increased, as evidenced by the increased amplitude of the trace pattern of the 3M lung (Figure 2O). Waveform analysis provided a quantitative assessment of lung function parameters to provide a functional correlation with the structural maturation of the postnatal lung. Progressive increases in tidal and minute volume were evident during postnatal lung development, increasing 8-fold $(0.012 \pm 0.001$ vs. $0.113 \pm$ $0.011, \mathrm{p}<0.001$; Figure 2P) and 10-fold (3.09 \pm 0.30 vs. 


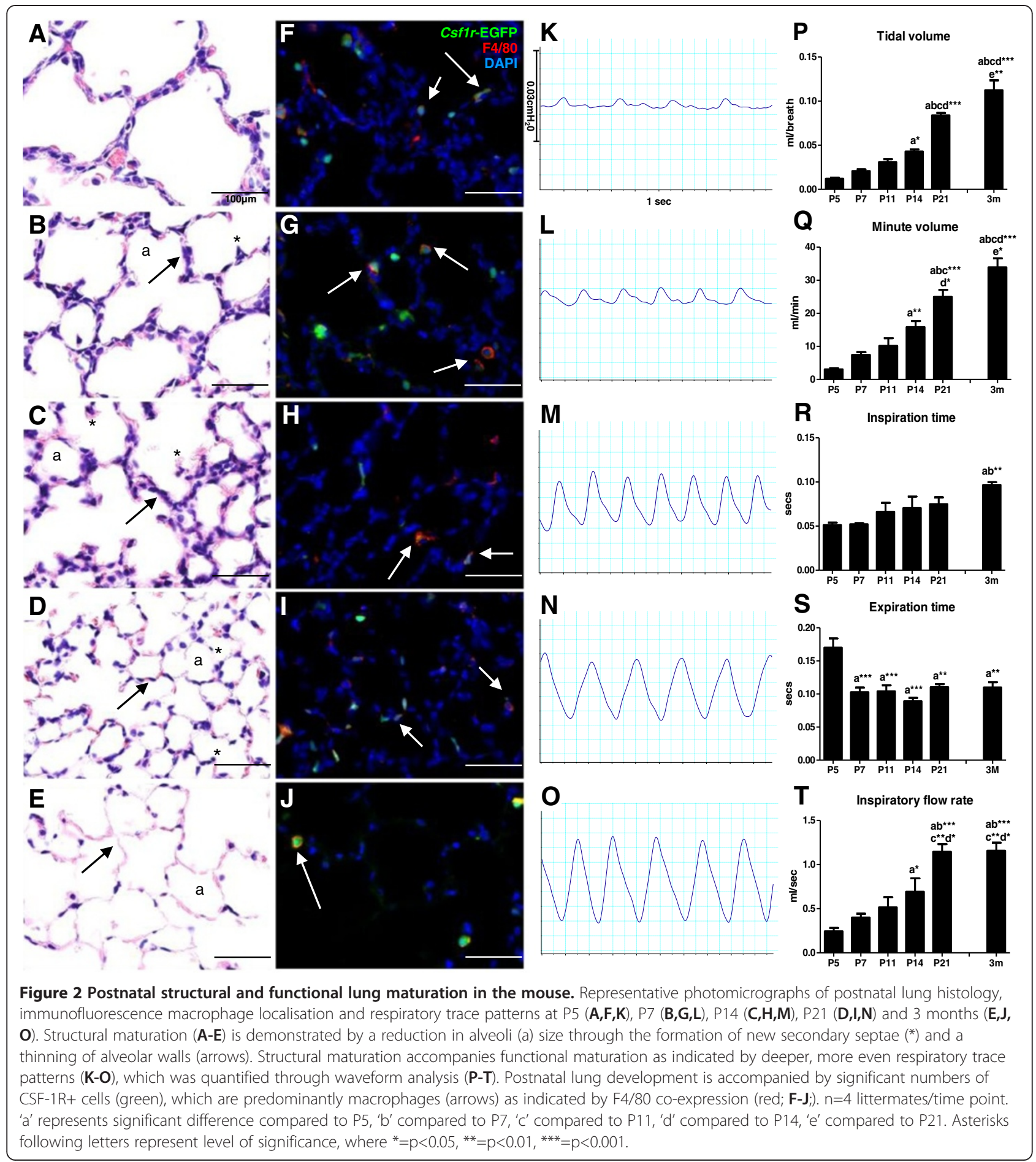

$33.93 \pm 2.70, \mathrm{p}<0.001$; Figure $2 \mathrm{Q}$ ) from P5 to $3 \mathrm{M}$, respectively, as the number of alveoli and overall size of the gas exchange compartment of the lung increased. As observed in the respiratory trace, breath patterns in immature lungs were quite uneven with short inhalations and slow dribbled exhalations at P5 and P7. This was also demonstrated in the waveform measurements, where expiration time decreased with the onset of alveolarisation (Figure 2S) and inspiration time progressively increased (Figure 2R) as a more even breath pattern emerged. A progressive increase in inspiratory flow rate was also observed, increasing approximately 4-fold 
from P5-P21 (0.24 \pm 0.04 vs. $1.15 \pm 0.09, \quad \mathrm{p}<0.001)$ when a maximum flow rate was then reached and maintained in the adult lung at 3M (Figure 2T).

\section{Lung macrophage number is increased during alveolarisation}

To more comprehensively assess the correlation between macrophages and alveolarisation, flow cytometry was performed to quantitatively examine the proportion and number of macrophages in the lung during postnatal development and into adulthood (Figure 3A-C). Again EGFP transgene expression facilitated the quantification of Csf1r+ myeloid cells (Figure 3D), which were confirmed as predominantly macrophages by consistent co-expression of F4/80 (Figure 2F-J) evident in both alveolar and interstitial macrophage subpopulations (Figure 3E\&F). Lungs were analysed at P1 in the saccular stage, at P5 when the lung transitions from the saccular to alveolarisation stage, at P7, P14 and P21 during alveolarisation, and at 3M in the mature lung. During postnatal life the overall cellularity of the lung gradually increased peaking at P14 during alveolarisation with an approximately 5 -fold increase compared to $\mathrm{P} 1\left(7.78 \pm 0.14 \times 10^{6}\right.$ vs. $35.50 \pm 5.01 \times 10^{6}$, $\mathrm{p}<0.001$; Figure $3 \mathrm{~A}$ ). From P14 onwards, as alveoli continued to develop and mature the total cellularity of the lung decreased. At 3M, despite the overall size difference, cellularity was comparable between the mature lung composed primarily of air sacs and the dense lung at P1 $\left(7.78 \pm 0.14 \times 10^{6}\right.$ vs. $\left.12.24 \pm 2.23 \times 10^{6}, \mathrm{p}=\mathrm{ns}\right)$. Similarly, the number of macrophages followed a parallel trend, and increased 9-fold from P1 to P14 (5.65 $\pm 0.87 \times 10^{5}$ vs. $\left.50.68 \pm 4.95 \times 10^{5}, \mathrm{p}<0.001\right)$, before a -2.5 -fold reduction by $3 \mathrm{M}\left(50.68 \pm 4.95 \times 10^{5}\right.$ vs. $19.93 \pm 1.39 \times 10^{5}$, $\mathrm{p}>0.05$; Figure $3 \mathrm{~B})$. In contrast to number, the proportion of macrophages in the lung steadily increased throughout postnatal life (Figure 3C). During the saccular stage at P1 and P5, macrophages represented approximately $8 \%$ of cells in the lung. This is significantly

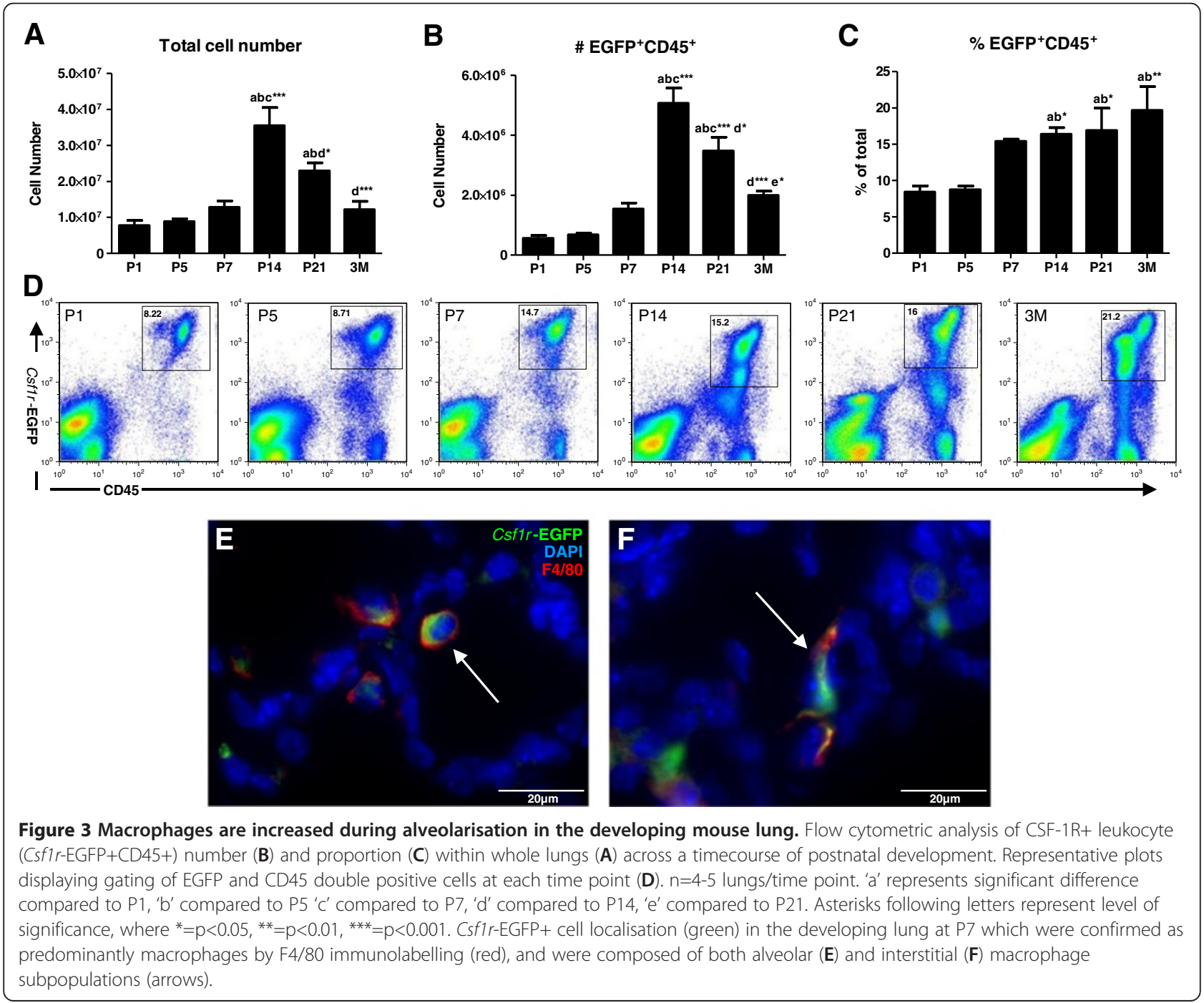


increased at P14 and P21 during alveolarisation to approximately $16 \%(\mathrm{p}<0.05)$, and at $3 \mathrm{M}$ a significant resident macrophage population was maintained in the adult lung (Figure 3C).

\section{Macrophages are polarised to an M2 phenotype during alveolarisation}

Correlations between macrophage phenotype and stages of lung development were investigated by analysing expression of genes indicative of different macrophage activation states (Figure 4). An upregulated expression of Ccl2, Nos 2 and Tnf is associated with an M1 or classical phenotype where macrophages contribute to host defence. M2 or alternatively activated macrophages are important in tissue remodelling, immunoregulatory and trophic functions, and are characterised by upregulation of genes including Arg1, Ccl17, Mrc1. A limited correlation between M1 gene expression and postnatal lung development was observed, although $\mathrm{Ccl} 2$ expression was highest after birth (Figure 4A) and Nos 2 decreased in later life at P21 and 3M (Figure 4B). In contrast, significant upregulation of M2 genes showed a distinct correlation with the key period of alveolar development. Arg1 expression was low in early postnatal life and began to increase at P14 (Figure 4D). At P21, Arg1 expression peaked and was 48 -fold higher than at P1 $(\mathrm{p}<0.01)$, before returning to a low level of expression in the adult lung. Ccl17 was also shown to increase during the alveolarisation stage (Figure 4E). After low expression from birth to P7, Ccl17 expression increased 9-fold at P14 $(\mathrm{p}<0.001)$ before decreasing by P21. The resident population of macrophages in the mature lung at $3 \mathrm{M}$ also maintained significant Ccl17 expression. Similarly, Mrc1 expression remained unchanged throughout early postnatal lung development but peaked at P14 with a 3-fold increase compared to P7 ( $<<0.01$; Figure 4F). Expression was decreased at P21 before high levels of Mrc1 expression were maintained in the resident lung macrophage population at $3 \mathrm{M}$. When all the genes analysed are presented on the same graph, the increase in the three genes indicative of an M2 macrophage phenotype is particularly evident (Figure 4G). This demonstrates a clear correlation between M2 macrophage phenotype and the key period of alveolarisation.

\section{CSF-1 administration increases developmental macrophages in the lung and is associated with increased Igf1 expression}

CSF-1 is the primary regulator of macrophage differentiation, survival and proliferation, and during development it plays an essential and non-redundant role in regulating organogenic macrophage functions $[17,18,33,34]$. Administration of CSF-1 to neonatal mice was shown to increase the number and proportion of developmental macrophages within the lung at P5. Flow cytometric analysis was performed on whole lungs, with populations of Csf1rEGFP+ leukocytes (Figure 5C) further gated on F4/80 expression to investigate macrophages (Figure 5D\&E). There was a trend towards an increase in total cellularity in CSF1 -treated lungs (Figure 5A). CSF-1 treatment resulted in a $6 \%$ increase in macrophage number $\left(58.10 \pm 2.49 \times 10^{4}\right.$ vs. $\left.69.32 \pm 2.56 \times 10^{4}, \mathrm{p}<0.05\right)$, and a $19 \%$ increase in macrophage proportion $(57.77 \pm 1.15 \%$ vs. $63.88 \pm 1.78 \%, \mathrm{p}<0.05)$, compared to PBS-treated littermates (Figure 5B). Analysis of gene expression also indicated that the CSF-1-mediated increase in macrophages was associated with upregulation of the Th2-associated molecule Fizz1 (1.05 \pm 0.18 vs. $1.56 \pm$ $0.05, \mathrm{p}<0.05$; Figure $5 \mathrm{~F}$ ) and the important growth regulator Igf1 (1.01 \pm 0.11 vs. $2.55 \pm 0.41, \mathrm{p}<0.05$; Figure 5G).

These effects of CSF-1 administration were confirmed to be via a macrophage-mediated mechanism as the CSF-1R was expressed exclusively on interstitial myeloid cells and not other cells of the developing lung. In situ hybridisation for the CSF-1R at E12.5 (Figure $5 \mathrm{H}$ ) and Csf1r driven EGFP transgene expression at P7 (Figure 5I) confirmed that the trophic activity of CSF-1 during lung development is through macrophage regulation. Furthermore, the finding that CSF-1 supplementation promoted a trophic M2 macrophage phenotype highlights CSF-1 and the manipulation of CSF-1-responsive cells as a potential intervention for rescuing or promoting organ development and maturation.

\section{Discussion}

The renewed interest in macrophages has stemmed from an increased understanding of monocyte/macrophage heterogeneity and how it relates to functional diversity [35-38]. Differential activation states have been broadly classified as M1, which encompasses macrophages involved in host defence and inflammation, and M2, which represent a more wound healing or tissue remodelling phenotype. Despite the M1/M2 activation dichotomy arising from studies of tissue disease and repair, understanding macrophage phenotype and function has implications for discerning and potentially enhancing their contribution to organ development. Functions of M2 macrophages, such as extracellular matrix (ECM) production, release of trophic factors and promotion of angiogenesis, are fundamental to organogenesis. Furthermore, our microarray expression profiling has revealed that embryonic macrophages in developing lungs, kidneys and brains show a comparable gene expression profile consistent with an M2 activation state [11]. In addition, CSF-1 can also promote an 'M2' macrophage activation state, which is increasingly being linked to tissue repair and regeneration $[2,39,40]$.

During embryonic development, macrophages are located abundantly within the embryo and are present in 


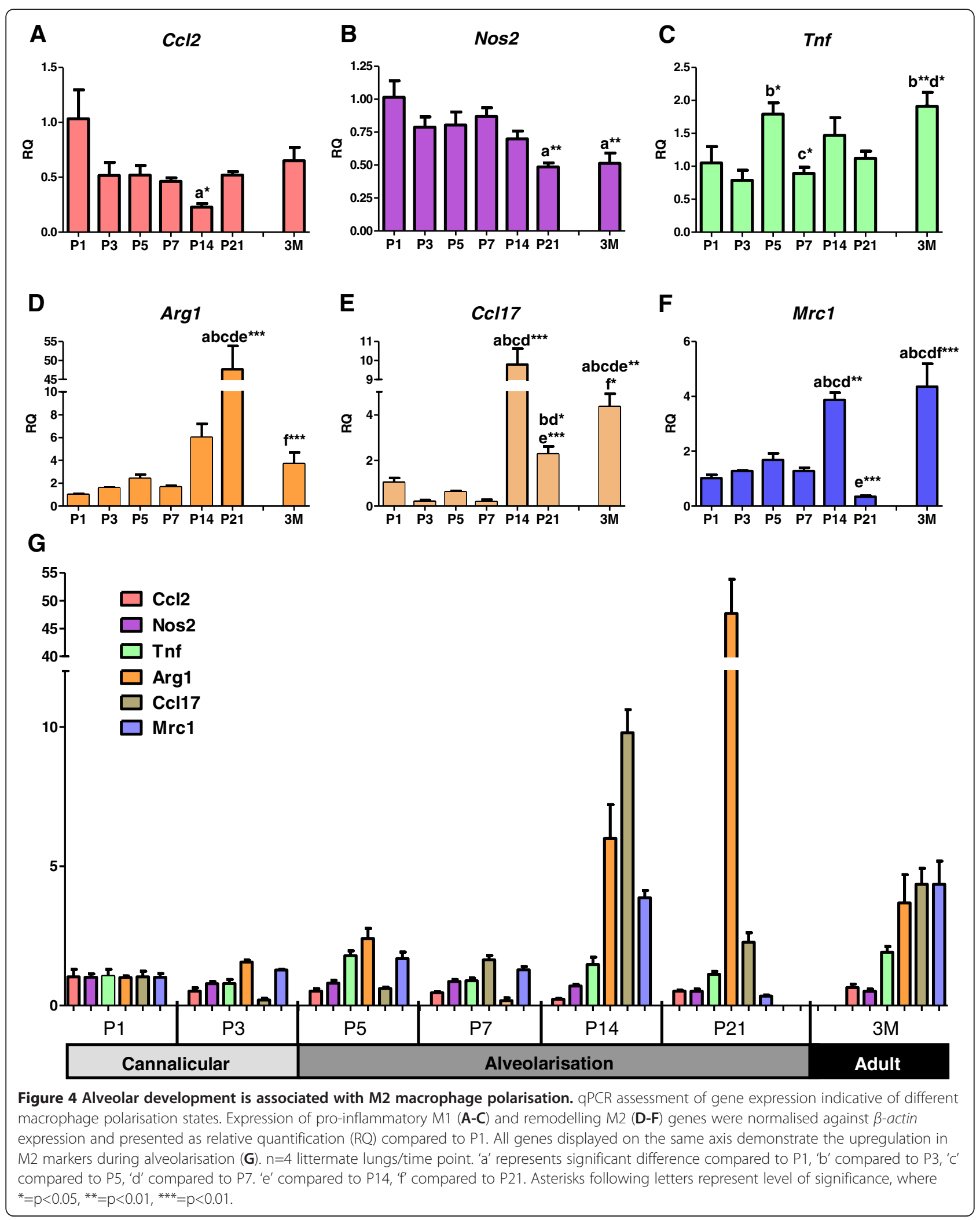



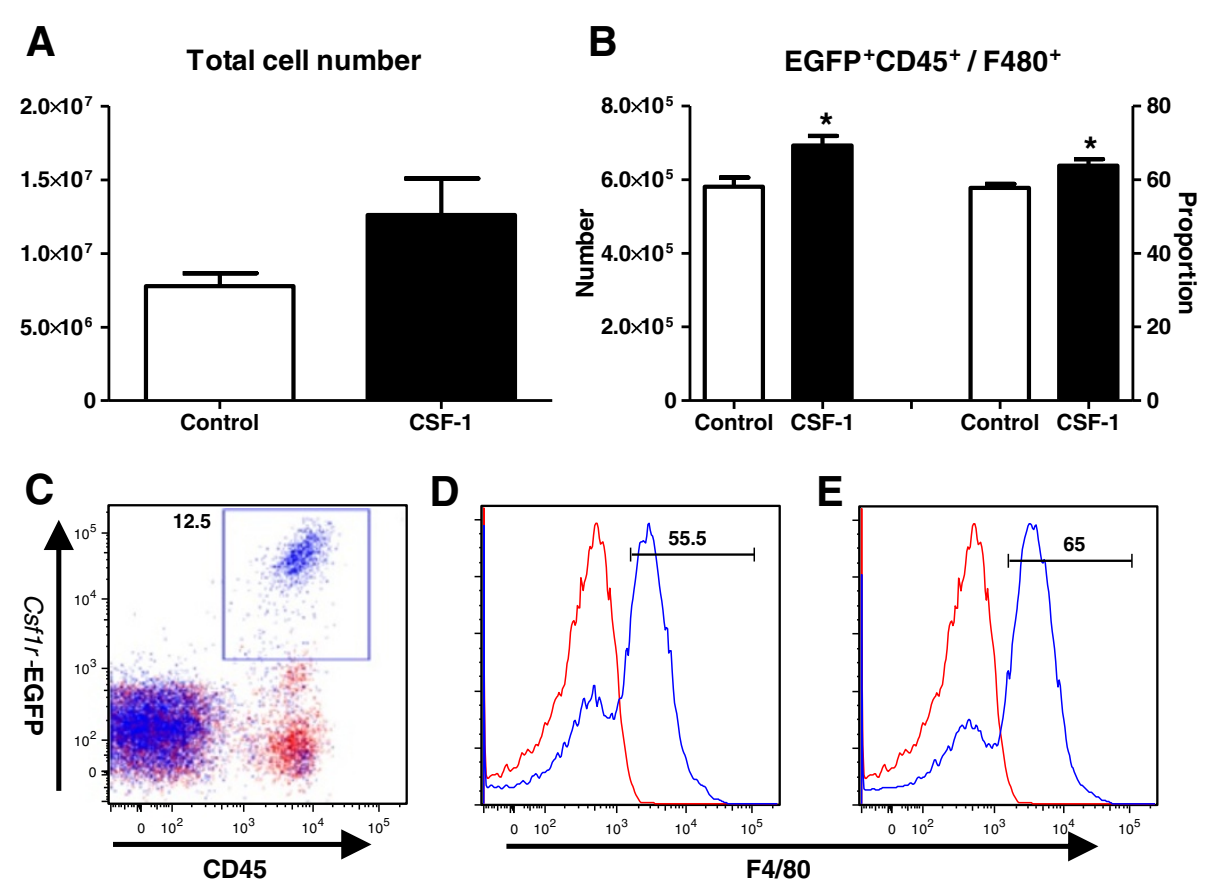

$\mathbf{F}$

Fizz1

G

lgf1
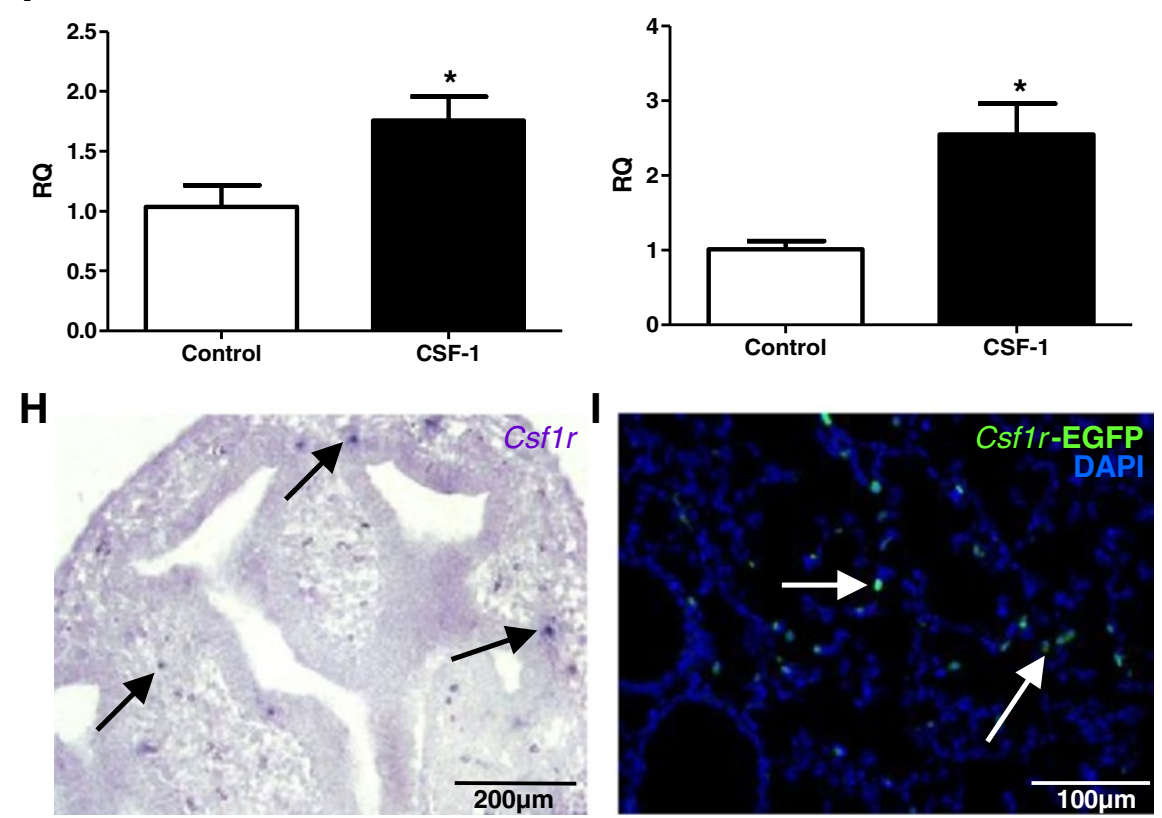

Figure 5 CSF-1 increases developmental macrophages and promotes M2 gene and IGF-1 upregulation. Flow cytometric analysis of macrophages $(\mathbf{A}-\mathbf{E})$ and $\mathrm{qPCR}$ analysis of gene expression in whole lungs $(\mathbf{F} \& \mathbf{G})$ at P5, following administration of mrCSF-1 (1 $\mu \mathrm{g} / \mathrm{g}$ i.p. at final

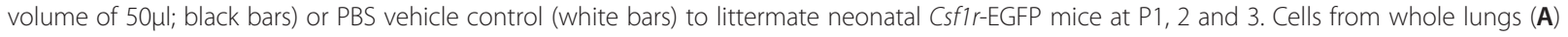
were gated on Csf1r-EGFP+CD45+ myeloid cells (C) and further gated on F4/80 expression to assess macrophage number and proportion (B), as displayed in representative histograms from control (D) and CSF-1-treated mice (E). Staining (blue) is overlayed with an isotype control (red). Igf1 (G) and $\mathrm{M} 2$ gene Fizz1 (F) expression was normalised against $\beta$-actin expression and presented as RQ compared to controls. $n=3-4$ littermate lungs/treatment. ${ }^{*}=\mathrm{p}<0.05$. Photomicrographs of in situ hybridisation for a Csf1r riboprobe at E12.5 (H; purple) and fluorescence Csf1r-EGFP transgene expression at P5 ( $\mathbf{I}$; green) demonstrated that during development the CSF-1R is expressed on interstitial myeloid cells (arrows), and not on the developing lung epithelium. 
virtually all developing organs [7]. Macrophage functions that support organogenesis include clearance of apoptotic cellular debris associated with tissue remodelling [41] and the provision of trophic support by producing a range of regulatory mediators [5,42]. Macrophages also contribute to appropriate cellular differentiation $[8,21,22]$ and angiogenic regulation [43], through both the production of angiogenic factors [44] and by physically directing angiogenic positioning [10]. As the lung buds form, macrophages surround the elongating primary bronchi [24]. The use of the Csf1r-EGFP reporter mice has been an important tool in demarcating the developmental role of macrophages in the embryo and has demonstrated a significant population of CSF-1R-expressing macrophages within the lungs at E13.5 [14]. Eliciting its effect through binding with the CSF-1R [45], CSF-1 is a pleiotropic growth factor also important in the regulation of pregnancy, fetal development and tissue regeneration [Reviewed in [33,34,40]].

Macrophages in the lung have been well described for their functions in host defence and inflammatory diseases, however the importance of CSF-1R+ macrophages in contributing to lung development has not been elucidated. The present study demonstrates the localisation of CSF-1R+EGFP+ alveolar and interstitial macrophages, which co-express the mature macrophage marker F4/80, in developing lungs during postnatal development. In addition, CSF-1R+ macrophages were identified in embryonic lung explants using wholemount immunofluorescence microscopy, where they were found to accumulate at branch points during lung branching morphogenesis.

The proposal that key organogenic periods are accompanied by an M2 macrophage phenotype was examined in the postnatal lung. During alveolarisation from P14 to P21, the expression of the M2 markers examined (Arg1, Ccl17 and Mrc1) showed a significantly increased expression. The remodelling functions of M2 macrophages are in accordance with the structural changes occurring within the lung at this time. Mrc1 provides an important mechanism for cellular clearance associated with homeostasis and tissue reorganisation [46]. Arg1 is associated with collagen formation and ECM production [47]. This study thus highlights the importance of macrophages in the alveolarisation stage of lung development, and in particular the association with an M2 activation state. Furthermore in the adult lung, an upregulation of M2 genes was also observed, supporting the homeostatic and immunomodulatory functions of resident pulmonary macrophages.

We have previously reported that CSF-1-responsive developmental macrophages are associated with growth and organ development, with delivery of recombinant protein to neonatal mice resulting in increased body and organ weight [2]. The present study showed that administration of CSF-1 to neonatal mice was also increased the number of macrophages in the developing lung and promoted an increase in Fizz1 (Retnla) and Igf1 expression. Fizz1 is an important mediator of lung development and maturation, and is upregulated during the saccular and alveolar stages, where its angiogenic and proliferative functions are suggested to promote alveolar development [48]. Fizz1 is also reported to participate in lung maturation by modulating surfactant production [49]. Expressed on lung cells such as mesenchymal and alveolar type II cells, the function of macrophage-derived Fizz1 is under-examined in previous reports, and its upregulation with CSF-1 supplementation indicates it may provide beneficial effects in regulating lung development.

The increase in the key growth regulator IGF-1 in response to CSF-1 administration provides insight regarding the potential mechanism of trophic macrophage function in organogenesis, and also supports an emerging link between CSF-1, macrophages and the IGF-1 growth axis [34]. Interestingly, many of the growth and developmental deficiencies observed in CSF-1-deficient mice are common to IGF-1-deficient animals [50]. Moreover, an interaction between CSF-1 and the IGF-1 growth axis is supported by the finding that CSF-1-deficient rats fail to produce the postnatal spike in IGF-1 [34]. Furthermore, IGF-1 production as a key mechanism of trophic macrophage function is supported by a previous study which demonstrated that kidney regeneration in an experimental model of acute kidney disease is mediated by CSF-1-responsive macrophages and an upregulation of IGF-1 [2]. This has important parallels for normal lung development as IGF-1 increases during alveolarisation, and the promotion of lung maturation using retinoic acid and dexamethasone has been shown to correlate positively with increased levels of IGF-1 [51].

Identification of M2-polarised macrophages as an important component of the organogenic milieu during alveolar development has important potential clinical implications, not only for understanding normal developmental processes, but also for addressing lung immaturity and the impact of neonatal inflammation of developmental perturbation. Inflammatory activation of macrophages not only contributes to tissue damage and perturbation of organ development through pro-inflammatory injury, but also disrupts morphogenesis in the lung and alters the expression of key genes important in lung development [23,52]. Nuclear factor kappa-light-chain-enhancer of activated B cells (NF- $\mathrm{kB}$ ) signalling in fetal macrophages upregulates pro-inflammatory mediators such as interleukin-1 $\beta$ and alters expression of Wnt7b, bone morphogenic protein-4 [23] and fibroblast growth factor-10 [52]. Given the correlation between the 
timing of lung structural establishment and lung immunological maturity [53], it is plausible that the developmental deficits associated with inflammation may also result from skewing of macrophages prematurely away from their organogenic activities toward proinflammatory mediation roles.

Understanding the regulation of alveolar development has particular relevance with regard to clinical implications of developmental perturbation, and especially in the setting of preterm birth. The neonatal mouse provides an excellent model for studying these aspects of lung development as mice are born at a stage where earlier aspects of organ development are still ongoing. The period of postnatal development in the mouse was characterised; correlating structural and functional maturation with macrophage localisation. The histological time course of postnatal lung development demonstrates the processes of structural maturation, whereby large saccules evident at the end of the saccular stage at P5 begin to subdivide through secondary septation to form definitive alveoli clearly evident at P21. This period of alveolarisation is critical in establishment of the gas exchange units required for proper function of the lung. This is evidenced by the chronic lung dysfunction associated with disruptions of alveolar development that often result from preterm birth and injurious therapeutic interventions that are required to keep the neonate alive $[54,55]$. Indeed, Mund et al. reported that murine alveolarisation occurs in two stages; phase 1 from P4-21 where alveoli arise from immature septae, and phase 2 from P21-36 where alveoli lift off from existing mature alveoli [32].

The histological time course of postnatal lung development was complemented by analysis of functional maturation over this time. Changes in breath cycle parameters were examined throughout postnatal lung development using unrestrained barometric whole-body plethysmography. This technique has been utilised in adult models of lung injury $[28,29]$ and was optimised for use in neonatal mice in our study. Modifications including the adjustment of the Perspex chamber size and increased sensitivity settings for pressure transduction recording enabled the measurement of lung function from as early as P5. Trace recordings showed discernable changes in breath patterns from P5 to adulthood, which when analysed provided quantitative changes in lung function. As the number of alveoli increased and the gas exchange compartment expanded, there was a correlation with changes in lung function parameters including an 8- and 10-fold increase in tidal and minute volume from the beginning of alveolarisation at P5 to adulthood. Normalisation of inspiratory flow rate and expiratory time were also observed over this time period of postnatal development as the chest wall and diaphragm mature.
The unique saccular architecture of the mature lung is associated with a different developmental cellularity pattern than that of solid organs. Overall cellularity increases rapidly in the alveolarisation phase and peaks at P14. From this stage, development is associated with significant remodelling and apoptosis as alveolar sacs form and mature, resulting in a decrease in cellularity in the adult mature lung. By digesting whole lungs, both interstitial as well as alveolar macrophages were able to be included for analysis, as opposed to the commonly used bronchoalveolar lavage-based collection method which restricts analysis to alveolar macrophages only. The proportion of macrophages in the lung is highest during the alveolarisation stage of lung development. A significant resident population is also maintained in the adult lung post completion of development, indicative of the unique air-exposed environment and the importance of pulmonary macrophages in clearing inhaled debris and modulating immune responses. The increased proportion of macrophages observed during alveolarisation - a time that lacks immunological relevance in this normal setting therefore suggests that macrophages are associated with developmental functions.

\section{Conclusion}

This study has demonstrated that macrophages provide a valuable contribution to normal lung development, and in particular that macrophages are increased and display an M2 polarisation phenotype during alveolarisation. An improved understanding of the organogenic environment important in regulating alveolar development has significant clinical relevance. The impact of inflammation and therapeutics on organogenic macrophage populations should be considered when studying the dysregulation and damage of the neonatal lung associated with preterm birth. It also supports research into modulation of macrophages in lung development to provide a novel intervention for enhancing lung maturation.

\section{Competing interests}

CVJ \& SDR hold a patent relating to the content of this manuscript

\section{Authors' contributions}

CVJ contributed to study design, performed the acquisition and analysis of data, and wrote the manuscript. TMW and SS assisted with flow cytometry, KAW participated in embryonic explant culture, HD assisted with plethysmography and lung histology, BAR performed in situ hybridisation under the supervision of MHL, GJ provided intellectual input and critical drafting of the manuscript and SDR oversaw study design and coordination, data interpretation and writing of the manuscript. All authors read and approved the final manuscript.

\section{Acknowledgments}

This work was supported by the National Health and Medical Research Council Project Grant \# 1003806 and Development Grant \# 1000615. HD is funded by an ARC Australian Post-Doctoral Research Fellowship. The authors wish to acknowledge Monash Micro Imaging, Monash University for technical support. 


\section{Author details}

'Department of Anatomy and Developmental Biology, Monash University, Clayton, Victoria, Australia. ${ }^{2}$ The Ritchie Centre, Monash Institute of Medical Research, Monash University, Clayton, Victoria, Australia. ${ }^{3}$ Institute for Molecular Bioscience, The University of Queensland, St Lucia, Brisbane Australia.

Received: 8 January 2013 Accepted: 28 March 2013

Published: 5 April 2013

\section{References}

1. Morrisey EE, Hogan BL: Preparing for the first breath: genetic and cellular mechanisms in lung development. Dev Cell 2010, 18:8-23.

2. Alikhan MA, Jones CV, Williams TM, Beckhouse AG, Fletcher AL, Kett MM, Sakkal S, Samuel CS, Ramsay RG, Deane JA, et al: Colony-stimulating factor1 promotes kidney growth and repair via alteration of macrophage responses. Am J Pathol 2011, 179:1243-1256.

3. Duffield JS, Forbes SJ, Constandinou CM, Clay S, Partolina M, Vuthoori S, Wu S, Lang R, Iredale JP: Selective depletion of macrophages reveals distinct, opposing roles during liver injury and repair. J Clin Invest 2005, 115:56-65.

4. Boulter L, Govaere O, Bird TG, Radulescu S, Ramachandran P, Pellicoro A, Ridgway RA, Seo SS, Spee B, Van Rooijen N, et al: Macrophage-derived Wnt opposes notch signaling to specify hepatic progenitor cell fate in chronic liver disease. Nat Med 2012, 18:572-579.

5. Lin SL, Li B, Rao S, Yeo EJ, Hudson TE, Nowlin BT, Pei H, Chen L, Zheng JJ, Carroll TJ, et al: Macrophage Wnt7b is critical for kidney repair and regeneration. Proc Natl Acad Sci U S A 2010, 107:4194-4199.

6. London A, Itskovich E, Benhar I, Kalchenko V, Mack M, Jung S, Schwartz M: Neuroprotection and progenitor cell renewal in the injured adult murine retina requires healing Monocyte-derived macrophages. J Exp Med 2011, 208:23-39.

7. Ovchinnikov DA: Macrophages in the embryo and beyond: much more than just giant phagocytes. Genesis 2008, 46:447-462.

8. Geutskens SB, Otonkoski T, Pulkkinen MA, Drexhage HA, Leenen PJ: Macrophages in the murine pancreas and their involvement in fetal endocrine development in vitro. J Leukoc Biol 2005, 78:845-852.

9. Ingman W, Wyckoff J, Gouon-Evans V, Condeelis J, Pollard JW: Macrophages promote collagen fibrillogenesis around terminal end buds of the developing mammary gland. Dev Dyn 2006, 235:3222-3229.

10. Fantin A, Vieira JM, Gestri G, Denti L, Schwarz Q, Prykhozhij S, Peri F, Wilson SW, Ruhrberg C: Tissue macrophages act as cellular chaperones for vascular anastomosis downstream of VEGF-mediated endothelial tip cell induction. Blood 2010, 116:829-840.

11. Rae F, Woods K, Sasmono T, Campanale N, Taylor D, Ovchinnikov DA Grimmond SM, Hume DA, Ricardo SD, Little MH: Characterisation and trophic functions of murine embryonic macrophages based upon the use of a Csf1r-EGFP transgene reporter. Dev Biol 2007, 308:232-246.

12. Lichanska AM, Hume DA: Origins and functions of phagocytes in the embryo. Exp Hematol 2000, 28:601-611.

13. Ovchinnikov DA, van Zuylen WJ, DeBats CE, Alexander KA, Kellie S, Hume DA: Expression of Gal4-dependent transgenes in cells of the mononuclear phagocyte system labeled with enhanced cyan fluorescent protein using Csf1r-Gal4VP16/UAS-ECFP double-transgenic mice. J Leukoc Biol 2008, 83:430-433.

14. Sasmono RT, Oceandy D, Pollard JW, Tong W, Pavli P, Wainwright BJ, Ostrowski MC, Himes SR, Hume DA: A macrophage colony-stimulating factor receptor-green fluorescent protein transgene is expressed throughout the mononuclear phagocyte system of the mouse. Blood 2003, 101:1155-1163.

15. Cecchini MG, Dominguez MG, Mocci S, Wetterwald A, Felix R, Fleisch $H$, Chisholm O, Hofstetter W, Pollard JW, Stanley ER: Role of colony stimulating factor- 1 in the establishment and regulation of tissue macrophages during postnatal development of the mouse. Development 1994, 120:1357-1372.

16. Stefater JA 3rd, Ren S, Lang RA, Duffield JS: Metchnikoff's Policemen: macrophages in development, homeostasis and regeneration. Trends $\mathrm{Mol}$ Med 2011, 17:743-752.

17. Wiktor-Jedrzejczak W, Bartocci A, Ferrante AW Jr, Ahmed-Ansari A, Sell KW, Pollard JW, Stanley ER: Total absence of colony-stimulating factor 1 in the macrophage-deficient osteopetrotic (op/op) mouse. Proc Natl Acad Sci U S A 1990, 87:4828-4832.
18. Yoshida H, Hayashi S, Kunisada T, Ogawa M, Nishikawa S, Okamura H, Sudo T, Shultz LD: The murine mutation osteopetrosis is in the coding region of the macrophage colony stimulating factor gene. Nature 1990, 345:442-444.

19. Dai X, Ryan G, Hapel A, Dominguez M, Russell R, Kapp S, Sylvestre V, Stanley E: Targeted disruption of the mouse colony-stimulating factor 1 receptor gene results in osteopetrosis, mononuclear phagocyte deficiency, increased primitive progenitor cell frequencies, and reproductive defects. Blood 2002, 99:111-120.

20. Gouon-Evans V, Rothenberg ME, Pollard JW: Postnatal mammary gland development requires macrophages and eosinophils. Development 2000 127:2269-2282.

21. Banaei-Bouchareb L, Gouon-Evans V, Samara-Boustani D, Castellotti MC, Czernichow P, Pollard JW, Polak M: Insulin cell mass is altered in Csf1op/ Csf1op macrophage-deficient mice. J Leukoc Biol 2004, 76:359-367.

22. Banaei-Bouchareb L, Peuchmaur M, Czernichow P, Polak M: A transient microenvironment loaded mainly with macrophages in the early developing human pancreas. J Endocrinol 2006, 188:467-480.

23. Blackwell TS, Hipps AN, Yamamoto Y, Han W, Barham WJ, Ostrowski MC, Yull FE, Prince LS: NF-kappaB signaling in fetal lung macrophages disrupts airway morphogenesis. J Immunol 2011, 187:2740-2747.

24. Higashi K, Naito M, Takeya M, Ando M, Araki S, Takahashi K: Ontogenetic development, differentiation, and phenotypic expression of macrophages in fetal rat lungs. J Leukoc Biol 1992, 51:444-454.

25. Li MO, Sarkisian MR, Mehal WZ, Rakic P, Flavell RA: Phosphatidylserine receptor is required for clearance of apoptotic cells. Science 2003, 302:1560-1563.

26. Wynes MW, Frankel SK, Riches DW: IL-4-induced macrophage-derived IGF-I protects myofibroblasts from apoptosis following growth factor withdrawal. J Leukoc Biol 2004, 76:1019-1027.

27. Rajagopal J, Carroll TJ, Guseh JS, Bores SA, Blank LJ, Anderson WJ, Yu J, Zhou Q, McMahon AP, Melton DA: Wnt7b Stimulates embryonic lung growth by coordinately increasing the replication of epithelium and mesenchyme. Development 2008, 135:1625-1634.

28. Milton $P L$, Dickinson $H$, Jenkin $G$, Lim R: Assessment of respiratory physiology of C57BL/6 mice following bleomycin administration using barometric plethysmography. Respiration 2011, 83:253-266.

29. Murphy S, Lim R, Dickinson H, Acharya R, Rosli S, Jenkin G, Wallace E: Human amnion epithelial cells prevent bleomycin-induced lung injury and preserve lung function. Cell Transplant 2010, 2010:2010.

30. Drorbaugh JE, Fenn WO: A barometric method for measuring ventilation in newborn infants. Pediatrics 1955, 16:81-87.

31. Rumballe B, Georgas K, Little MH: High-throughput paraffin section in situ hybridization and dual immunohistochemistry on mouse tissues. CSH Protoc 2008 2008. pdb prot5030.

32. Mund SI, Stampanoni M, Schittny JC: Developmental alveolarization of the mouse lung. Dev Dyn 2008, 237:2108-2116.

33. Douglass TG, Driggers L, Zhang JG, Hoa N, Delgado C, Williams CC, Dan Q, Sanchez R, Jeffes EW, Wepsic HT, et al: Macrophage colony stimulating factor: not just for macrophages anymore! a gateway into complex biologies. Int Immunopharmacol 2008, 8:1354-1376.

34. Gow DJ, Sester DP, Hume DA: CSF-1, IGF-1, and the control of postnatal growth and development. J Leukoc Biol 2010, 88:475-481.

35. Martinez FO, Sica A, Mantovani A, Locati M: Macrophage activation and polarization. Front Biosci 2008, 13:453-461

36. Mosser DM, Edwards JP: Exploring the full spectrum of macrophage activation. Nat Rev Immunol 2008, 8:958-969.

37. Williams TM, Little MH, Ricardo SD: Macrophages in renal development injury, and repair. Semin Nephrol 2010, 30:255-267.

38. Pollard JW: Trophic macrophages in development and disease. Nat ReV Immunol 2009, 9:259-270.

39. Zhang MZ, Yao B, Yang S, Jiang L, Wang S, Fan X, Yin H, Wong K, Miyazawa T, Chen J, et al: CSF-1 signaling mediates recovery from acute kidney injury. J Clin Invest 2012, 122:4519-4532.

40. Hamilton JA: Colony-stimulating factors in inflammation and autoimmunity. Nat Rev Immunol 2008, 8:533-544.

41. Lang RA, Bishop JM: Macrophages are required for cell death and tissue remodeling in the developing mouse eye. Cell 1993, 74:453-462.

42. Nacu N, Luzina IG, Highsmith K, Lockatell V, Pochetuhen K, Cooper ZA Gillmeister MP, Todd NW, Atamas SP: Macrophages produce TGF-beta-induced (beta-ig-h3) following ingestion of apoptotic cells and regulate MMP14 levels and collagen turnover in fibroblasts. J Immunol 2008, 180:5036-5044. 
43. Nucera S, Biziato D, De Palma M: The interplay between macrophages and angiogenesis in development, tissue injury and regeneration. Int J Dev Biol 2011, 55:495-503.

44. Stefater JA 3rd, Lewkowich I, Rao S, Mariggi G, Carpenter AC, Burr AR, Fan J, Ajima R, Molkentin JD, Williams BO, et al: Regulation of angiogenesis by a non-canonical Wnt-Flt1 pathway in myeloid cells. Nature 2011, 474:511-515.

45. Sherr CJ: Colony-stimulating factor-1 receptor. Blood 1990, 75:1-12.

46. Allavena P, Chieppa M, Monti P, Piemonti L: From pattern recognition receptor to regulator of homeostasis: the double-faced macrophage mannose receptor. Crit Rev Immunol 2004, 24:179-192.

47. Durante W, Johnson FK, Johnson RA: Arginase: a critical regulator of nitric oxide synthesis and vascular function. Clin Exp Pharmacol Physiol 2007, 34:906-911.

48. Wagner KF, Hellberg AK, Balenger S, Depping R, Dodd OJ, Johns RA, Li D: Hypoxia-induced mitogenic factor has antiapoptotic action and is upregulated in the developing lung: coexpression with hypoxiainducible factor-2alpha. Am J Respir Cell Mol Biol 2004, 31:276-282.

49. Tong $Q$, Zheng L, Dodd-o J, Langer J, Wang D, Li D: Hypoxia-induced mitogenic factor modulates surfactant protein $B$ and $C$ expression in mouse lung. Am J Resp Cell Mol 2006, 34:28-38.

50. Liu JL, Yakar S, LeRoith D: Conditional knockout of mouse insulin-like growth factor-1 gene using the Cre/loxP system. Proc Soc Exp Biol Med 2000, 223:344-351.

51. Liu H, Chang L, Rong Z, Zhu H, Zhang Q, Chen H, Li W: Association of insulin-like growth factors with lung development in neonatal rats. J Huazhong Univ Sci Technolog Med Sci 2004, 24:162-165.

52. Benjamin JT, Carver BJ, Plosa EJ, Yamamoto Y, Miller JD, Liu JH, van der Meer R, Blackwell TS, Prince LS: NF-kappaB activation limits airway branching through inhibition of Sp1-mediated fibroblast growth factor10 expression. J Immunol 2010, 185:4896-4903.

53. Dickie R, Tasat DR, Alanis EF, Delfosse V, Tsuda A: Age-dependent changes in porcine alveolar macrophage function during the postnatal period of alveolarization. Dev Comp Immunol 2009, 33:145-151.

54. Moss TJ: Respiratory consequences of preterm birth. Clin Exp Pharmacol Physiol 2006, 33:280-284

55. Kinsella JP, Greenough A, Abman SH: Bronchopulmonary dysplasia. Lancet 2006, 367:1421-1431.

doi:10.1186/1465-9921-14-41

Cite this article as: Jones et al:: M2 macrophage polarisation is associated with alveolar formation during postnatal lung development.

Respiratory Research 2013 14:41.

\section{Submit your next manuscript to BioMed Central and take full advantage of:}

- Convenient online submission

- Thorough peer review

- No space constraints or color figure charges

- Immediate publication on acceptance

- Inclusion in PubMed, CAS, Scopus and Google Scholar

- Research which is freely available for redistribution 